Association for Information Systems AIS Electronic Library (AISeL)

Wirtschaftsinformatik Proceedings 2001

Wirtschaftsinformatik

September 2001

\title{
IuK-Standort Bayern - Erfolgsfaktoren im internationalen Wettbewerb
}

Erwin Huber

Bayerische Staatskanzlei, staatsminister@stk.bayern.de

Follow this and additional works at: http://aisel.aisnet.org/wi2001

\section{Recommended Citation}

Huber, Erwin, "IuK-Standort Bayern - Erfolgsfaktoren im internationalen Wettbewerb" (2001). Wirtschaftsinformatik Proceedings 2001. 1.

http://aisel.aisnet.org/wi2001/1

This material is brought to you by the Wirtschaftsinformatik at AIS Electronic Library (AISeL). It has been accepted for inclusion in Wirtschaftsinformatik Proceedings 2001 by an authorized administrator of AIS Electronic Library (AISeL). For more information, please contact elibrary@aisnet.org. 
In: Buhl, Hans Ulrich, u.a. (Hg.) 2001. Information Age Economy; 5. Internationale Tagung Wirtschaftsinformatik 2001. Heidelberg: Physica-Verlag

ISBN: 3-7908-1427-X

(C) Physica-Verlag Heidelberg 2001 


\section{IuK-Standort Bayern - Erfolgsfaktoren im internationalen Wettbewerb}

\section{Erwin Huber}

Staatsminister und Leiter der Bayerischen Staatskanzlei

Die Messlatte für Erfolg liegt hoch in der IuK-Spitzentechnologie. Der Wettbewerb ist hochkarätig und international. Die Innovationszyklen in dieser Schlüsselbranche des 21. Jahrhunderts werden immer kürzer. Schonfristen in der Konfrontation gibt es nicht - weder mit den "alten großen" noch mit den "jungen kleinen" IuK-Unternehmen.

Wer als Unternehmer Trendsetter in der IuK-Branche sein will, für den sind Innovationsfähigkeit, Geschwindigkeit und Risikobereitschaft ebenso unverzichtbar wie Kreativität und Flexibilität. Wer in der Politik Motor für die IuK-Branche sein will, der kann zwar unternehmerische Innovationen nicht erfinden, aber der kann ein Klima gestalten für eine Kultur der Selbstständigkeit und der Eigeninitiative, für zukunftssichere Arbeitsplätze und für die internationale Wettbewerbsfähigkeit.

Die Bayerische Staatsregierung hat früher als andere auf eine konsequente Modernisierung in Forschung, Wissenschaft und durch die Technologien der Zukunft gesetzt. Diese offensive und zielstrebige Politik des Fortschritts wirkt als TurboMotor für die IuK-Branche. Der Freistaat gilt heute als der bedeutendste Standort der Informations- und Kommunikationstechnologie auf dem europäischen Kontinent. Bayern ist im Wettbewerb bestens positioniert.

Kein anderes Bundesland und keine andere Region in Europa beheimatet so viele große Namen aus der IT-Welt wie Bayern: Apple, Cisco, Compaq, Datev, Intel, Lotus, Lucent, Microsoft, Oracle, Siemens, Siemens Fujitsu, Sun Microsystems, Toshiba, Viag Interkom, Yahoo!, 3Com und viele andere haben im Freistaat ihre Welt-, Europa- oder Deutschlandzentrale.

Auch bei den jungen IT-Unternehmen nimmt Bayern eine Spitzenstellung ein. Viele Start-Ups wie Atrada, Consors und SuSE in Nürnberg, openshop in NeuUlm, DCI in Starnberg, Ciao.com und Secaron in München betreiben ihre Geschäfte von Bayern aus. Bereits heute siedeln $30 \%$ der deutschen IuK-Start-Ups in Bayern. Auf dem Neuen Markt stammen allein $30 \%$ der Firmen aus dem Freistaat. Noch deutlicher ist die Spitzenposition Münchens und Bayerns bei der Finanzierung von Start-Ups. Nach einer Untersuchung von Bain \& Company vom März diesen Jahres fließt nahezu jede zweite Mark, die bundesweit in E-BusinessGründungen investiert wird, nach Bayern.

Nach Auffassung der renommierten Unternehmensberatungsgesellschaft Boston Consulting Group zählt die Region München inzwischen zu den weltweit interessantesten High-Tech-Standorten. Vor München rangiert nur noch das Silicon Val- 
ley, Boston und Tel Aviv. Als einzige deutsche und europäische Region kommt München damit unter die Top Five-Standorte der Welt.

Neben dem IuK-Mekka München sind das Städtedreieck Nürnberg-Fürth-Erlangen, Landshut, Augsburg, Aschaffenburg, Würzburg und Regensburg weitere ITCluster von internationaler Bedeutung.

Unsere Politik für den Technologiestandort Bayern ist eine erfolgreiche Politik für mehr Beschäftigung. Nach einer Erhebung von McKinsey hatte sich Bayern bereits bis 1997 zur mit Abstand bedeutendsten IuK-Region Deutschlands entwickelt. Der Raum München war dabei mit über 70.000 Beschäftigten in Deutschland der größte IuK-Standort, in Europa der zweitgrößte und weltweit die Nummer 4 nach dem Silicon Valley und den Großräumen Boston und London.

Inzwischen hat „Cybavaria“, wie man in der Start-Up-Szene sagt, seine Position in der internationalen Spitzengruppe weiter ausgebaut. Nach einer Untersuchung der Industrie- und Handelskammern Nürnberg und München planen die IuK-Unternehmen in München bis zum Jahr 2004 die Aufstockung ihrer Beschäftigtenzahl um weitere 80.000 auf dann 235.000. Die Unternehmen in Nürnberg rechnen im gleichen Zeitraum mit einer Steigerung um 23.000 auf 74.000. Damit hätten sich die Beschäftigtenzahlen in München innerhalb von 7 Jahren mehr als verdreifacht und in Nürnberg fast verfünffacht.

Die Bedeutung Bayerns im IuK-Bereich kann man auch daran messen, dass bei einem Bevölkerungsanteil von $15 \%$ in Deutschland über $36 \%$ der Beschäftigten der deutschen Computerfertigung in Bayern tätig sind, bei elektronischen Bauelementen sind es $39 \%$ und bei elektronischen Medien sogar $40 \%$.

In der dynamischen IuK-Branche wäre Stillstand bereits Rückschritt. Gerade in einer Zeit, in der die Euphorie der ersten Jahre einer gewissen Ernüchterung gewichen ist, müssen wir die Nutzung und Effizienz der Informationstechnologien und vor allem des Internet weiter steigern. Lassen wir uns durch den Börsencrash am Neuen Markt nicht verunsichern. Ich halte dies für eine natürliche Marktbereinigung nach einem Gründerboom, wie sie in der Wirtschaftsgeschichte immer wieder zu beobachten war. Der neue Realismus ist eine gute Basis für die Zukunft. Jetzt heißt es: Von den Besten lernen! Hinfallen und Niederschläge sind keine Schande. Die Kraft zum Aufstehen ist entscheidend!

Der Freistaat Bayern ist Kraftfeld und Magnet im IuK-Wettbewerb, und wir bauen unsere Stärken durch eine wirtschafts- und innovationsfreundliche Politik gezielt weiter aus. Nur Standorte, die dauerhaft international hervorstechen, können langfristig eigene Talente halten und einen kontinuierlichen Zustrom der Weltbesten sichern. Standortvorteile auf hohem Niveau nur zu halten - das wäre im globalen Wettbewerb viel zu wenig.

Die Erfolge Bayerns im internationalen Vergleich kommen nicht von ungefähr. Mit der "Offensive Zukunft Bayern" und der "High-Tech-Offensive" mit einem Gesamtvolumen von 8,25 Milliarden DM sichern wir den wirtschaftlichen und so- 
zialen, aber auch den ökologischen und kulturellen Wohlstand in allen Regionen Bayerns. Bayern liegt mit einem Aufwand für Forschung und Entwicklung von 2,8\% - gemessen am Bruttoinlandsprodukt - weltweit in der Spitzengruppe und übertrifft den Bundesdurchschnitt von 2,3\% bei weitem. Fast ein Viertel aller deutschen Patentanmeldungen kommt aus Bayern.

Wir vereinen die richtige Mischung an Qualifikationen in unserem Land: Know how, Köpfe, Kapital - ein Netzwerk der Kompetenz aus erstens hochqualifizierten Fachkräften durch die Nähe zu renommierten Universitäten und Forschungseinrichtungen, zweitens Unternehmern und drittens Kapitalgebern.

Von im wahrsten Sinne des Wortes grundlegender Bedeutung für den IuK-Standort Bayern ist eine entsprechende Aus- und Weiterbildung der Menschen in unserem Land.

An den bayerischen Schulen wird die Ausstattung mit Computern und Internetzugang energisch vorangetrieben. Heute verfügen rund $99 \%$ der Gymnasien, $98 \%$ der Fach- und Berufsoberschulen, $94 \%$ der Wirtschaftsschulen und $93 \%$ der Berufs- und Realschulen über einen Netzzugang. In einer breit angelegten Fortbildungskampagne qualifizieren wir alle 70.000 Lehrer an den 3.000 weiterführenden bayerischen Schulen für den Einsatz der neuen Medien und Informationstechnologien im Unterricht. Zur Verbesserung des Einsatzes der Informations- und Kommunikationstechniken an den Schulen werden allein durch den Staat 60 Mio. DM eingesetzt, hinzu kommen hohe Beiträge der kommunalen Schulaufwandsträger.

Bayern hat eine exzellente Hochschullandschaft mit 11 Universitäten und 17 Fachhochschulen. Derzeit studieren in Bayern über 60.000 Studenten die Fächer Mathematik, Informatik, Natur- und Ingenieurswissenschaften. Mit 60 Millionen DM aus Mitteln der High-Tech-Offensive erhöhen wir die Kapazitäten für Studienanfänger des Fachs Informatik an den bayerischen Universitäten und Fachhochschulen um $20 \%$. Bis 2007 wollen wir die Zahl der Absolventen in den Fächern der Informationstechnologien um 50\% vergrößern. Wir setzen beim Thema Nachwuchsmangel auf das Motto: Ausbildung im Inland hat Vorrang!

Neben den Universitäten und Fachhochschulen genießen auch die in der IuKTechnik tätigen Fraunhofer-Institute in München und Erlangen und die landesweiten Forschungsverbünde einen hervorragenden Ruf.

Auf und neben dem Campus von Universitäten werden Technologie- und Gründerzentren als "Brutkästen" für junge Unternehmen eingerichtet. Die Staatsregierung unterstützt den leichteren Übergang von der Uni zur selbstständigen Existenz zum Beispiel durch das Förderprogramm FLÜGGE, durch Existenzgründerzentren oder Venture-Capital.

Zudem stärken die Virtuelle Hochschule Bayern und der Virtuelle Campus den IT-Standort Bayern. Mit der Virtuellen Hochschule Bayern werden die besten Online-Angebote bayerischer Hochschullehrer den bayerischen Studenten gebündelt 
zur Verfügung gestellt. Als erste Verbundeinrichtung in Deutschland ist sie im Mai 2000 ans Netz gegangen. Sie greift auf das multimediale Angebot und Wissen aller Hochschulen unseres Landes zurück. Der Schwerpunkt des Lehrangebots liegt derzeit auf den Fächergruppen Informatik, Ingenieurswissenschaften, Medizin und Wirtschaftswissenschaften. Mit 2500 registrierten Studenten zum Sommersemester diesen Jahres hat sich die Zahl seit dem Take-off der Virtuellen Hochschule bereits verfünffacht

Wir fördern den engen Dialog und den Wissenstransfer zwischen Wissenschaft und Wirtschaft einerseits und Politik andererseits, z. B. im WissenschaftlichTechnischen Beirat oder im Internet-Beirat der Bayerischen Staatsregierung, im Industriebeirat des Wirtschaftsministeriums oder im Münchner Kreis.

Der Aufstieg Bayerns zu einem der weltweiten Zentren der Informationstechnologie wäre nicht möglich gewesen ohne eine frühzeitige Förderung dieser Branche durch die Staatsregierung. Der Freistaat hat mit „BayernOnline“ schon im Jahr 1994 als erstes deutsches Bundesland auf die neuen Informations- und Kommunikationstechnologien gesetzt - und damit in- und ausländischen Investoren ein positives Signal gegeben in Zeiten, als andernorts der Computer noch als ,Jobkiller Nummer 1" mit Argwohn betrachtet wurde.

Durch eine Anschubfinanzierung in Höhe von 148 Millionen DM aus Privatisierungserlösen für insgesamt 53 Pilotprojekte hat die Bayerische Staatsregierung im Rahmen der IuK-Initiative „Bayern-Online“ ein Projektvolumen von über 500 Millionen DM ausgelöst. Die Projekte erstrecken sich auf die Bereiche Telekommunikationsinfrastruktur, Ländlicher Raum, Bildung, Behörden, Polizei, Verkehr, Medizin, mittelständische Wirtschaft und Digitaler Rundfunk. Wir haben nicht nur eine leistungsfähige Infrastruktur aufgebaut, sondern vor allem bei Betrieben und Bürgern Anreize geschaffen, die neuen Techniken und das Internet praktisch zu nutzen.

Schon damals waren die Ziele von „Bayern-Online“ wegweisend für die IuKBranche: Uns liegt daran, dass möglichst viele Bürger im Freistaat Kompetenz im Umgang mit moderner IuK-Technologie erlangen. Wir wollen eine ZweiklassenInformationsgesellschaft verhindern, in der ein digitaler Graben die „Online-Profis“ von den „Online-Analphabeten“ trennt. Das ist nicht zuletzt ja auch ein großer Bildungsauftrag. Medien- und Informationskompetenz sind die Schlüsselwörter der Zukunft.

Ein weiteres Ziel von „Bayern-Online“ war die Stärkung der ländlichen Regionen im Flächenstaat Bayern durch den Ausgleich von Standortnachteilen mittels moderner Telekommunikation. Neue Möglichkeiten der IuK-Technologien werden seither auch zur Leistungsverbesserung der Verwaltung genutzt. Und schließlich galt unser Augenmerk frühzeitig der Förderung des Einsatzes der IuK-Technologie in kleinen und mittelständischen Unternehmen, um diese für den Wettbewerb zu stärken und neue bisher nicht erreichbare Märkte über das Internet zu öffnen. 
Das Bayernnetz können Bürger sowie mittelständische Unternehmen unentgeltliche und nicht-kommerziell nutzen. Dadurch wurde die Gründung von BürgernetzVereinen angeregt, die wohnortnahe Zugänge zum Bayernnetz errichteten und regionale Informationen in das Internet einstellten. Bayern hat hierdurch die höchste Internet-Nutzerdichte und die größte Zahl an Internetprovidern in Deutschland erreicht.

Im Juli 2001 fand zum vierten Mal der BayernOnline-Kongress statt. Der Kongress hat das Ziel, die technologiebedingte gesellschaftliche Entwicklung zur Wissensgesellschaft im Freistaat Bayern jährlich zu dokumentieren und voranzutreiben. Kongresse wie BayernOnline in München und Fachmessen wie die neue eprocure in Nürnberg wirken als Katalysatoren und Multiplikatoren des Fortschritts. Sie sind wichtige nationale und internationale Schaufenster der Zukunft.

Um die Attraktivität des Internet für Bürger und Unternehmen aus der mittelständischen Wirtschaft erneut zu steigern, hat der Freistaat Bayern ein weiteres bundesweites Pilotprojekt angestoßen - den „Virtuellen Marktplatz Bayern“. Die elektronische Handelsplattform des Virtuellen Marktplatzes enthält inzwischen über zehn regionale Marktplätze, daneben den zentralen Teil des Behördenwegweisers und ein Unternehmensverzeichnis, in dem alle bayerischen Betriebe mit einem kostenlosen Grundeintrag vertreten sind. Im Gegensatz zu anderen - für die Nutzer oft unübersichtlichen Internetangeboten wie Internet-Shops und -Malls bietet der Virtuelle Marktplatz Bayern ein überzeugendes Produkt für Bayern mit den drei Säulen E-Government, E-Commerce und Events. Mit Hilfe dieses integrierten Marktplatzes wollen wir die Nutzung des Internets durch die Bürger, die Wirtschaft und die öffentliche Verwaltung erneut stimulieren.

BayernOnline wird ergänzt und fortgeführt durch die High-Tech-Offensive mit einem Gesamtvolumen von 2,65 Mrd. DM aus Privatisierungserlösen. Dabei macht die IuK-Technik mit rund 500 Mio. DM einen bedeutenden Schwerpunkt aus.

Die Software-Offensive - Teil der High-Tech-Offensive - umfasst Maßnahmen für IuK-Kompetenz und Qualifikation, für die angewandte Softwareforschung, für Unternehmensgründungen im IuK-Bereich und für den Softwareeinsatz in Mittelstand und Handwerk. Wir wissen: Nur Spitzenergebnisse in Forschung und Entwicklung schaffen die Grundlage für weltweit erfolgreiche Produkte

Ein wichtiger Standortvorteil im IuK-Wettbewerb ist auch der schnelle Zugang zu wichtigen Kunden. Der Freistaat weist eine starke Konzentration an IuK-Anwendern auf. So ist München Versicherungsplatz Nummer 1 in Deutschland und einer der größten Versicherungsplätze der Welt sowie Bankenplatz Nr. 2 in Deutschland. Daneben ist Bayern zentraler Standort für die Elektroindustrie, für Medienunternehmen, die Luft- und Raumfahrtindustrie, die Mikroelektronik, den Automobilbau mit seinen zahlreichen Zulieferern, den Maschinenbau (CAD-Systeme) sowie eine Vielzahl mittelständischer Unternehmen in unterschiedlichsten Branchen. Old und new economy ergänzen sich in Bayern auf hervorragende Weise. 
In Bayern existiert eine stetig wachsende Venture-Capital-Szene. Etwa ein Fünftel des in Deutschland investierten Venture Capitals fließt in die Region München. Mehr als 40 Risikokapitalgesellschaften sind dort ansässig. Die meisten dieser Fonds sind aktive Investoren in Life Siences und Informations- und Kommunikationstechnologien.

Um die Chancen neuer Technologieunternehmen in Bayern zu verbessern, hat die Staatsregierung 1995 die Bayern Kapitalgesellschaft mit Sitz in Landshut gegründet. Weitere finanzielle Hilfe und Beratung für junge Technologieunternehmen bietet das Bayerische Programm zur Förderung technologieorientierter Unternehmensgründungen BayTOU und die Projektbegleitung junger Firmen durch Technologie- und Gründerzentren, die die Staatsregierung fördert. Neu eingerichtet wurde ein Seed-Capital-Fonds in einer Höhe von 28 Millionen für die Frühphase von Existenzgründungen in der Hochtechnologie.

Zur Unterstützung der Ansiedlung von IuK- und Medien-Unternehmen in Bayern und zur Präsentation von Erfolgen hat der Freistaat eine Agentur für Medien, Informations- und Kommunikationstechnologie gegründet. Der Slogan der Agentur heißt „GotoBavaria“ - Nomen est omen! Ansiedlungswilligen Unternehmen und Existenzgründern aus dem Medien- und IuK-Bereich stehen umfassende Serviceangebote zur Verfügung. Die Agentur mit Hauptsitz in München nutzt auch das Büro des Freistaats in Palo Alto im Silicon-Valley als wichtige Kontaktstelle in den Hauptmarkt USA. Ein weiteres Büro für Standortmarketing wird in Bangalore/Indien eröffnet.

In München und in Nordbayern führt die Bayerische Staatsregierung bereits Businessplan-Wettbewerbe durch, die auf lebhaftes Interesse stoßen. Mit diesen Wettbewerben, die auf alle Regierungsbezirke ausgedehnt werden, fördern wir Gründermut und Eigeninitiative in allen Regionen Bayerns.

Die genannten Beispiel zeigen: Die Staatsregierung investiert ganz gezielt

- in den Ausbau der Kompetenz der Menschen in den Zukunftsbranchen,

- in zukunftsweisende Unternehmen und Unternehmensgründer,

- in eine gute finanzielle Infrastruktur.

Die Attraktivität des Standortes Bayern für Investoren macht schließlich auch Bayerns zentrale Lage in Europa in Verbindung mit der vorhandenen exzellenten Verkehrsinfrastruktur und der hohen Lebensqualität in unserem Land aus. Attraktive Kulturangebote und die schöne Naturlandschaft sind Anreize des Standorts Bayern. Wer viel arbeitet, der braucht eben auch Inspiration und der muss auftanken können in der Natur.

Bayern befindet sich auf IuK-Erfolgskurs. Unsere Innovationspolitik ist kein Selbstzweck, sondern hilft, Brücken in die Zukunft zu bauen für Wachstum, für neue Arbeitsplätze und damit für den sozialen Wohlstand in Bayern. 